\title{
The Effects of Multiple Sets of Squats and Jump Squats on Mechanical Variables
}

Brandon W. Snyder, Michael L. Rossetti, Gavin L. Moir, Shawn N. Munford, Shala E. Davis, FACSM. East Stroudsburg University of Pennsylvania, East Stroudsburg, PA.

PURPOSE: To investigate the effects of two non-ballistic squat and two ballistic jump squat protocols performed over multiple sets on mechanical variables. METHODS: In a counterbalanced cross-over design, 11 resistance-trained men (age: $21.9 \pm 1.8$ years; height: 1.79 $\pm 0.05 \mathrm{~m}$; mass: $87.0 \pm 7.4 \mathrm{~kg}$ ) attended four testing sessions during a three week period where they performed multiple sets of squats and jump squats with a load equivalent to $30 \% 1$-repeititon maximum under one of the following conditions: 1) three sets of four non-ballistic repetitions $(30 \mathrm{~N}-\mathrm{B}), 2)$ three sets of four non-ballistic repetitions with a 3-second pause between the eccentric and concentric phases $(30 \mathrm{PN}-\mathrm{B}), 3)$ three sets of four ballistic repetitions $(30 \mathrm{~B}), 4)$ three sets of four ballistic repetitions with a 3 -second pause between the eccentric and concentric phases (30PB). Force plates and a 3-D motion analysis system were used to determine the mean vertical velocity, mean vertical force, and mean power output during each repetition. RESULTS: Significantly greater vertical velocity (mean difference $[\mathrm{MD}]: 0.25-0.21 \mathrm{~m} / \mathrm{s}, \mathrm{p}<0.001$, effect size [ES]: $1.70-1.74$ ) and vertical force (MD: $526 \mathrm{~N}, \mathrm{p}<0.001$, ES: 1.61) were attained during 30B compared to $30 \mathrm{~N}-\mathrm{B}$ and $30 \mathrm{PN}-\mathrm{B}$. Vertical velocity during $30 \mathrm{~PB}$ was significantly greater than that attained during both $30 \mathrm{~N}-\mathrm{B}$ and $30 \mathrm{PN}-\mathrm{B}$ (MD: $0.21-0.23 \mathrm{~m} / \mathrm{s}, \mathrm{p}<0.001$, ES: $1.89-1.76$ ), and vertical force during 30PB was also significantly greater than that during 30N-B (MD: $478 \mathrm{~N}$, $\mathrm{p}<0.001$, ES: 1.63) and 30PN-B (MD: $478 \mathrm{~N}, \mathrm{p}<0.001$, ES: 1.62). Mean power output was significantly greater during 30B compared to both 30N-B (MD: $843 \mathrm{~W}, \mathrm{p}<0.0001$, ES: 1.66) and 30PN-B (MD: $869 \mathrm{~W}, \mathrm{p}<0.001$, ES: 1.67). Mean power output during 30PB was also significantly greater than that during $30 \mathrm{~N}-\mathrm{B}$ (MD: $711 \mathrm{~W}, \mathrm{p}<0.001$, ES: 1.73) and 30PN-B (MD: $737 \mathrm{~W}$, $\mathrm{p}<0.001$, ES: 1.72). The increase in power output across the three sets in the 30B condition was significantly greater than the small changes in power output observed during the $30 \mathrm{~N}-\mathrm{B}$ and $30 \mathrm{PN}-$ $\mathrm{B}$ conditions $(\mathrm{p}<0.001)$ and significantly different from the decrease observed during the 30PB condition between sets 2 and $3(p=0.015)$. CONCLUSION: Ballistic exercises are likely to be effective in the development of power output and their use across multiple sets may induce postactivation potentiation. 